\title{
Preventing Infectious Complications of Immunomodulation in COVID-19 in Foreign-Born Patients
}

\author{
Amir M. Mohareb ${ }^{1,2}$. Jacob M. Rosenberg ${ }^{1,2,3} \cdot$ Roby P. Bhattacharyya $^{1,2} \cdot$ Camille N. Kotton $^{1,2}$. \\ Jacqueline T. Chu ${ }^{1,2} \cdot$ Nikolaus Jilg $^{1,2} \cdot$ Kristen M. Hysell ${ }^{1,2} \cdot$ John S. Albin ${ }^{1,2} \cdot$ Pritha Sen $^{1,2} \cdot$ Seth M. Bloom ${ }^{1,2,3}$. \\ Abigail E. Schiff ${ }^{2} \cdot$ Kimon C. Zachary $^{1} \cdot$ Alyssa R. Letourneau ${ }^{1,2} \cdot$ Arthur Y. Kim $^{1,2} \cdot$ Rocio M. Hurtado $^{1,2,4}$
}

Accepted: 1 June 2021 / Published online: 22 June 2021

(c) The Author(s), under exclusive licence to Springer Science+Business Media, LLC, part of Springer Nature 2021

\begin{abstract}
Immunomodulating therapies for COVID-19 may carry risks of reactivating latent infections in foreign-born people. We conducted a rapid review of infection-related complications of immunomodulatory therapies for COVID-19. We convened a committee of specialists to formulate a screening and management strategy for latent infections in our setting. Dexamethasone, used in severe COVID-19, is associated with reactivation of latent tuberculosis, hepatitis B, and dissemination/ hyperinfection of Strongyloides species and should prompt screening and/ or empiric treatment in appropriate epidemiologic contexts. Other immunomodulators used in COVID-19 may also increase risk, including interleukin-6 receptor antagonist (e.g., tocilizumab) and kinase inhibitors. People with specific risk factors should also be screened for HIV, Chagas disease, and endemic mycoses. Racial and ethnic minorities in North America, including foreign-born persons, who receive immunomodulating agents for COVID-19 may be at risk for reactivation of latent infections. We develop a screening and management pathway for such patients.
\end{abstract}

Keywords COVID-19 · Inequity · Tuberculosis · Hepatitis B virus · Strongyloidiasis · Immigrant $\cdot$ Refugee $\cdot$ Health disparities $\cdot$ Chagas disease $\cdot$ HIV

\section{Background}

The death toll from COVID-19 continues to rise across the U.S. and around the world. In the U.S., in-hospital mortality for COVID-19 surpasses $10 \%$ among patients above the age of 50 years, and many patients require prolonged mechanical ventilation [1]. Racial and ethnic minorities bear a disproportionate burden of COVID-19 [2]. To alter the natural history of disease, clinicians have repurposed

Amir M. Mohareb and Jacob M. Rosenberg contributed equally to this work.

Amir M. Mohareb

amohareb@mgh.harvard.edu

1 Division of Infectious Diseases, Massachusetts General Hospital, 55 Fruit Street, Boston, MA 02114, USA

2 Harvard Medical School, Boston, MA, USA

3 Ragon Institute of MGH, MIT, and Harvard, Boston, MA, USA

4 Global Health Committee, Boston, MA, USA immunomodulatory drugs for use in hospitalized patients. Dexamethasone has become standard of care for patients with COVID-19 who require mechanical ventilation or supplemental oxygen, and biologic immunomodulators are the subject of ongoing investigation [3]. While immunomodulators hold promise for improving outcomes and preventing severe complications, they come with the risk of reactivation of latent infections, including tuberculosis, hepatitis B virus (HBV), Strongyloides stercoralis, and other infections. Populations at highest risk for these complications include foreign-born persons and racial/ethnic minorities. Our objective was to formulate an adaptable management strategy to mitigate the infection-related risks of immunomodulation in COVID-19 in these vulnerable populations.

\section{Methods}

We searched Pubmed with a search strategy that combined common latent infections in foreign-born patients (tuberculosis, HBV, Strongyloidiasis, Chagas disease, and endemic 
mycoses) and pharmacological agents being considered for experimental and off-label use in COVID-19. We searched an online dashboard of COVID-19 clinical trials (https:// www.covid-trials.org/) to determine immunomodulators being considered in COVID-19 management: corticosteroids, IL-6 signaling inhibitors (tocilizumab, sarilumab, sirukumab, siltuximab), and kinase inhibitors (ruxolitinib, tofacitinib, baricitinib, ibrutinib, acalabrutinib, and others). We convened a committee of Infectious Diseases specialists in our institution to establish protocols of screening, monitoring, prevention, and treatment of infection-related complications of immunomodulation used in COVID-19. With input from specialists from Oncology, Rheumatology, and Pulmonary and Critical Care, we developed a practical clinical algorithm for use by non-ID specialists for screening and monitoring for reactivation of latent infections in the course of COVID-19 treatment during this pandemic. We focused our final recommendations on the most important infections in foreign-born persons seen in our clinical setting.

\section{Results and Commentary}

The spectrum of disease in COVID-19 varies widely across populations. Early observations illuminated the role of the inflammatory cascade in the development of severe disease and critical illness [4]. Dexamethasone reduces 28-day mortality among patients with COVID-19 on mechanical ventilation or supplementary oxygen [3] and is thus frequently used in hospitalized patients. Among other pathways, specific consideration has been given to inhibition of IL-1, IL-6, and kinase signaling pathways [4]. An online database of COVID-19 clinical trials reported more than 80 clinical trials of IL-6 receptor antagonists and more than 45 trials of Janus Kinase (JAK)-inhibitors. While no consensus has yet been reached for clinical practice as of this review, recent evidence suggests benefit of IL- 6 receptor antagonism in certain patients [5]. Immunomodulation is also being used to modify the long-term natural history of severe COVID-19 among survivors following recovery from the acute phase of illness, as cases of long-term pulmonary fibrosis and restrictive lung disease are increasingly common [6].

Clinicians treating COVID-19 with immunomodulation must be prepared for the risk of infection-related complications. In the U.S., the COVID-19 outbreak has had a disproportionate effect on racial and ethnic minorities, including foreign-born persons [2]. These disparities may be rooted in differences in socioeconomic status and ability to engage in social distancing, combined with higher rates of crowded housing, employment in economic sectors designated as "essential work," and untreated cardiovascular and metabolic comorbidities. Populations disproportionately susceptible to severe COVID-19 overlap with those at higher risk of latent or opportunistic infections, namely HIV, tuberculosis, HBV, Strongyloidiasis, and Chagas disease, among others.

Clinicians caring for patients with COVID-19 may not be familiar with the potential for infection-related complications. Clinicians frequently miss screening for latent infections, even in patients with well-defined risk factors, prior to initiating immunomodulatory therapy for autoimmune diseases or cancer [7]. Among patients presenting with COVID-19 who do not have regular contact with the healthcare system, HIV screening should be performed. Without explicitly acknowledging the increased risk of latent infections in persons from minority communities, estimating this risk as accurately as possible, and considering mitigation strategies, clinical trials could disproportionately and inequitably exclude these groups from clinical trials of immunomodulation. At-risk communities could thus suffer a "double-hit" with a convergence of increased susceptibility to COVID-19 and decreased representation in clinical trials. Indeed, many effective biomedical advances have historically exacerbated existing inequity by means of unequal access. It is thus important to consider latent infections as potential drivers of unequal outcomes, access to clinical trials, or complications from experimental therapies, especially in the context of immunomodulation.

What is the risk of reactivation of latent infections associated with the use of immunomodulatory therapies for COVID-19? Unfortunately, there is a relative paucity of data to inform this question. Corticosteroids are wellknown to increase the risk of active tuberculosis (TB), and risk increases both with steroid dose and duration [8]. Patients infected with non-tuberculous mycobacteria may experience dissemination or worsening disease while on steroid therapy. The strong association of tuberculosis and non-tuberculous mycobacteria infection with TNF-inhibitors is well-described, and tuberculosis has been described with IL-6, IL-1, and JAK inhibitor therapies. Immunosuppression can provoke reactivation of chronic HBV infection, which can result in hepatic injury, and in some cases, acute liver failure leading to transplantation or death. The risk of HBV reactivation varies by the immunosuppressive agent and its duration of use. HBV reactivation has been reported in up to $5 \%$ of patients with resolved infection (HBV core antibody positivity with or without HBV surface antibody) receiving IL-6 antagonists for rheumatologic indications, and higher rates of reactivation are observed among those with detectable HBV surface antigen (HBsAg). This risk can be mitigated by active monitoring of HBV DNA, with or without initiation of anti-viral drugs, such as entecavir, tenofovir disoproxil fumarate, or tenofovir alafenamide. Strongyloides hyperinfection is a devastating and well-described complication of glucocorticoids and other immunosuppression in the setting of transplantation; its relative risk with novel immunomodulators is unknown, but cases of Strongyloides 
hyperinfection in COVID-19 immunomodulation are emerging $[9,10]$. Chagas disease, or American trypanosomiasis, is a vector-borne disease that is underdiagnosed in patients originating from Central and South America where it is endemic and present in some parts of the U.S. Chagas disease can cause a variety of long-term, multi-system complications. Immunosuppression may lead to more fulminant and morbid presentations in this patient population.

It is unknown whether infection risk with biologic agents, typically studied in the setting of rheumatologic diseases, can be extrapolated to the context of COVID-19. Use of biologic immunomodulating agents in rheumatic disease typically involves several years of use as a component of other immunosuppressing regimens in the outpatient setting. It is possible that the infection risks associated with immunomodulation in COVID-19 in the acute illness context could more closely resemble those in cytokine release syndrome, for example. It is also unknown if COVID-19 itself, even in the absence of pharmacologic immunomodulation, could predispose to reactivation of latent infections. When they do occur, reactivation of latent infections are difficult to diagnose in patients with prolonged hospitalization and complex medical problems related to COVID-19. Pulmonary and extra-pulmonary tuberculosis infection could be erroneously attributed to end organ effects of COVID-19. Clinicians without a high suspicion for HBV reactivation may miss this diagnosis since aminotransferase elevation is present in most hospitalized patients with COVID-19. Strongyloides hyperinfection and complications related to Chagas disease may also be missed in the setting of a complicated COVID-19 ICU hospitalization [9].

We formulated a management pathway for clinicians in our institution considering immunomodulation in patients with severe COVID-19 (Fig. 1; see Supplementary Table for references). These recommendations range from individualized management (tuberculosis, HBV, Chagas disease) to empiric treatment (ivermectin for Strongyloides infection [9]), with more detailed guidance that is regularly updated available at our website: https://www.massgeneral.org/news/ coronavirus/treatment-guidance/inpatient-care-recommenda tions\#treatment

Longer term follow-up of the recovering post-COVID population via primary health care and ambulatory settings is also a part of this initiative, as some of these potential infection-related complications may occur after the acute phase of illness has resolved. Longer term surveillance for these infections following COVID-19 hospitalization also provides a valuable opportunity to strengthen the linkage to care of patients who may have had limited access to primary care prior to the pandemic. Recognizing the absence of high-quality evidence to motivate clinical practice guidelines at this time, and the knowledge that clinicians in some parts of the nation may have less experience with these novel agents in at-risk populations, we believe these algorithms can provide useful guidance to others during the pandemic. These algorithms will require frequent updates as more is learned about COVID-19 and the effects of specific novel therapeutic agents. 


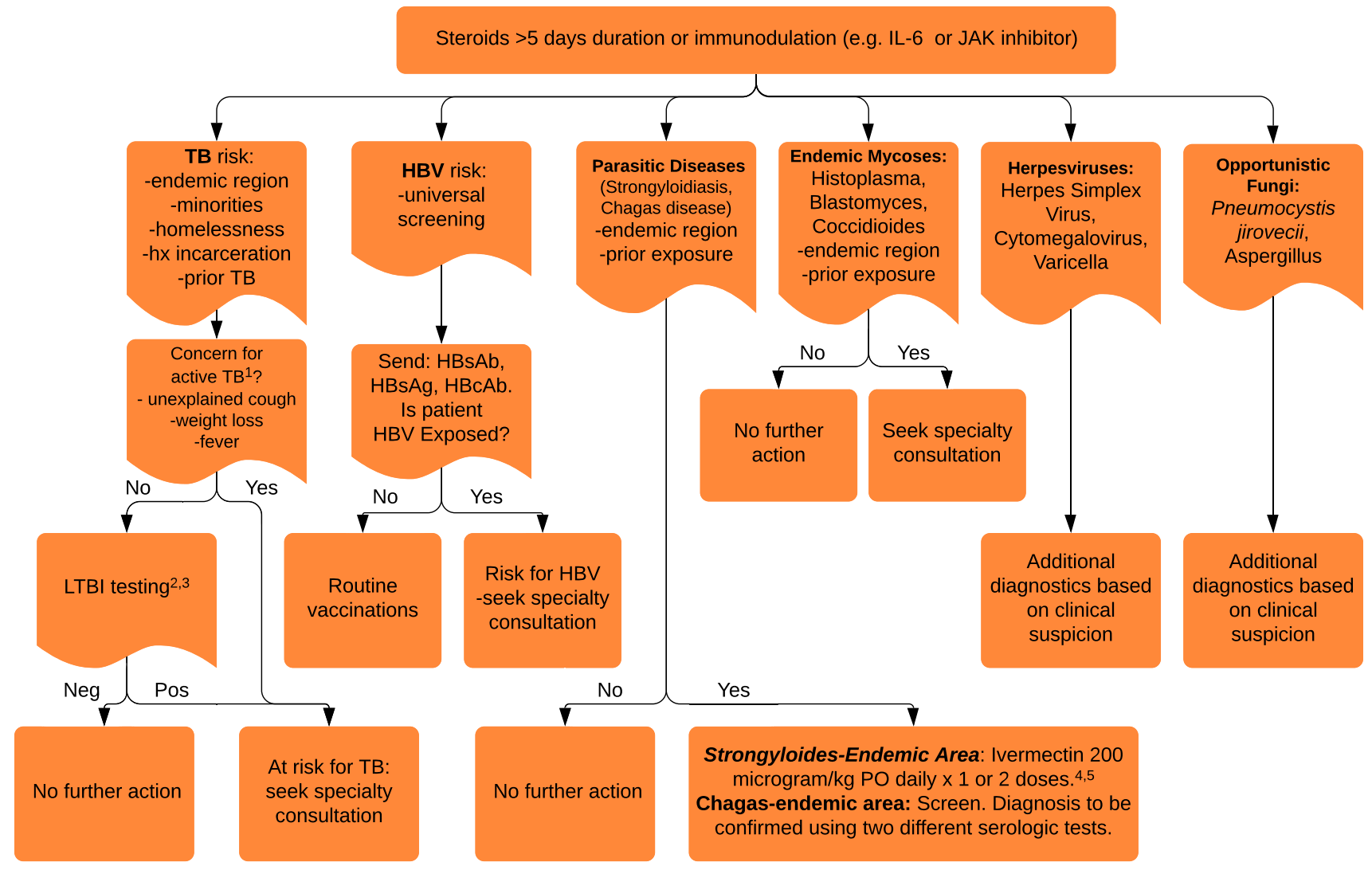

Fig. 1 Screening, monitoring, and treatment of reactivation of latent infections in the course of immunomodulatory treatment of COVID19. Note: $T B$ tuberculosis, $H B V$ hepatitis B virus, $L T B I$ latent TB infection, $H B s A b \mathrm{HBV}$ surface antibody, $H B s A g$ HBV surface antigen, $H B c A b$ total HBV core antibody. 1. These symptoms should precede the onset of acute COVID-19 symptoms by at least one month and not be otherwise explained by comorbid conditions. 2 . For patients with prior history of TB, LTBI testing is not indicated. 3. A positive LTBI test does not preclude use of immunomodulatory therapy with specialist input. 4. Strongyloides can reactivate with less than one week of steroid therapy, so in the appropriate host, we recommend prescribing ivermectin regardless of steroid duration. 5 .

\section{Conclusions}

The COVID-19 pandemic in the United States has deepened existing disparities in clinical outcomes for racial and ethnic minorities, including foreign-born patients. Recognizing and addressing the risk for infectious complications in atrisk populations is a critical component of addressing these disparities and ensuring safe access to immunomodulatory therapy for people of all communities.

Supplementary Information The online version contains supplementary material available at https://doi.org/10.1007/s10903-021-01225-4.
In settings where serologic testing with rapid turn-around time for Strongyloides is available and follow up can be ensured, serologic testing can be conducted prior to treatment; otherwise, empiric ivermectin treatment is recommended. Ivermectin can preferentially be dosed at $200 \mu \mathrm{g} / \mathrm{kg} \mathrm{PO} \times 2$ doses 14 days apart if follow-up dosing can be ensured. Many cases of uncomplicated Strongyloides infection may be treated with a single dose. Patients from West/Central Africa may be at risk for high-titer co-infection with other filarial nematodes, including Loa Loa, which is a contraindication to ivermectin administration. Clinicians should seek specialty consultation in these cases

Funding AMM is supported by the National Institutes of Health (NIH) [T32-AI007433]. JMR is supported by the NIH [T32-AI007387]. JSA is supported by NIH [T32-AI007061] and the Cystic Fibrosis Foundation. The NIH and the Cystic Fibrosis Foundation had no role in the design or authorship of this publication. The article contents are solely the responsibility of the authors and do not necessarily represent the official views of the NIH or the Cystic Fibrosis Foundation.

\section{Declaration}

Conflict of interest AYK has served on a scientific advisory board for Biomarin, Inc. JTC has the following conflicts of interest: stock in Pfizer, Johnson \& Johnson, Cisco Systems Inc and Boston Scientific Corp. All other authors have no other potential conflicts of interest to disclose. 


\section{References}

1. Richardson S, et al. Presenting characteristics, comorbidities, and outcomes among 5700 patients hospitalized with COVID-19 in the New York City Area. JAMA. 2020;323(20):2052.

2. Rossen LM, et al. Excess deaths associated with COVID-19, by age and race and ethnicity-United States, January 26-October 3, 2020. MMWR Morb Mortal Wkly Rep. 2020;69(42):1522-7.

3. RECOVERY Collaborative Group, et al. Dexamethasone in hospitalized patients with Covid-19. N Engl J Med. 2021;384:693-704.

4. Moore JB, June CH. Cytokine release syndrome in severe COVID19. Science. 2020;368(6490):473-4.

5. REMAP-CAP Investigators, et al. Interleukin-6 receptor antagonists in critically ill patients with Covid-19. N Engl J Med. 2021;384:1491-502.

6. George PM, et al. Pulmonary fibrosis and COVID-19: the potential role for antifibrotic therapy. Lancet Respir Med. 2020;8(8):807-15.
7. Hwang JP, et al. Low rates of hepatitis B virus screening at the onset of chemotherapy. J Oncol Pract. 2012;8(4):e32-9.

8. Youssef $\mathbf{J}$, et al. Infection risk and safety of corticosteroid use. Rheum Dis Clin N Am. 2016;42(1):157-76.

9. Stauffer WM, et al. COVID-19 and dexamethasone: a potential strategy to avoid steroid-related strongyloides hyperinfection. JAMA. 2020;324(7):623.

10. Lier AJ, et al. Case report: disseminated strongyloidiasis in a patient with COVID-19. Am J Trop Med Hyg. 2020;103(4):1590-2.

Publisher's Note Springer Nature remains neutral with regard to jurisdictional claims in published maps and institutional affiliations. 